\title{
Sensor-Derived Physical Activity Parameters Can Predict Future Falls in People with Dementia
}

\author{
Michael Schwenk ${ }^{\mathrm{a}-\mathrm{c}}$ Klaus Hauer $^{c}$ Tania Zieschang $^{c}$ Stefan Englert $^{\mathrm{d}}$ \\ Jane Mohler ${ }^{a, b}$ Bijan Najafi ${ }^{a}$ b
}

anterdisciplinary Consortium on Advanced Motion Performance (iCAMP), Department of Surgery, College of

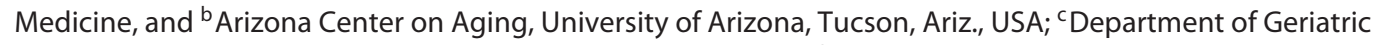
Research, Agaplesion Bethanien Hospital/Geriatric Centre, and d Institute of Medical Biometry and Informatics, University of Heidelberg, Heidelberg, Germany

\section{Key Words}

Fall prediction · Body-worn sensor · Physical activity ·

Dementia

\begin{abstract}
Background: There is a need for simple clinical tools that can objectively assess the fall risk in people with dementia. Wearable sensors seem to have the potential for fall prediction; however, there has been limited work performed in this important area. Objective: To explore the validity of sensorderived physical activity (PA) parameters for predicting future falls in people with dementia. To compare sensor-based fall risk assessment with conventional fall risk measures. Methods: This was a cohort study of people with confirmed dementia discharged from a geriatric rehabilitation ward. PA was quantified using 24-hour motion-sensor monitoring at the beginning of the study. PA parameters (percentage of walking, standing, sitting, and lying; duration of single walking, standing, and sitting bouts) were extracted using specific algorithms. Conventional assessment included performance-based tests (Timed Up and Go Test, Performance-Oriented Mobility Assessment, 5-chair stand) and questionnaires (cognition, ADL status, fear of falling, depression, previous faller). Outcome measures were fallers (at least one fall
\end{abstract}

in the 3-month follow-up period) versus non-fallers. Results: 77 people were included in the study (age $81.8 \pm 6.3$; community-dwelling $88 \%$, institutionalized $12 \%)$. Surprisingly, fallers and non-fallers did not differ on any conventional assessment ( $p=0.069-0.991)$, except for 'previous faller' $(p=$ 0.006). Interestingly, several PA parameters discriminated between the groups. The 'walking bout average duration', 'longest walking bout duration' and 'walking bout duration variability' were lower in fallers, compared to non-fallers $(p=0.008-0.027)$. The 'standing bout average duration' was higher in fallers $(p=0.050)$. Two variables, 'walking bout average duration' [odds ratio (OR) $0.79, p=0.012$ ] and 'previous faller' (OR 4.44, $p=0.007$ ) were identified as independent predictors for falls. The OR for a 'walking bout average duration' $<15 \mathrm{~s}$ for predicting fallers was $6.30(p=0.020)$. Combining 'walking bout average duration' and 'previous faller' improved fall prediction (OR 7.71, $p<0.001$, sensitivity/specificity $72 \% / 76 \%)$. Discussion: Results demonstrate that sensor-derived PA parameters are independent predictors of the fall risk and may have higher diagnostic accuracy in persons with dementia compared to conventional fall risk measures. Our findings highlight the potential of telemonitoring technology for estimating the fall risk. Results should be confirmed in a larger study and by measuring PA over a longer period of time.

\section{KARGER}

() 2014 S. Karger AG, Basel

0304-324X/14/0606-0483\$39.50/0

E-Mail karger@karger.com

www.karger.com/ger
Michael Schwenk, PhD

Interdisciplinary Consortium on Advanced Motion Performance (iCAMP)

Department of Surgery, College of Medicine, University of Arizona

1656 E Mabel Street, Tucson, AZ 85724 (USA)

E-Mail mschwenk@surgery.arizona.edu 


\section{Introduction}

Falls are a significant cause of injuries, loss of confidence, institutionalization and mortality in all older people $[1,2]$, but particularly in those with dementia $[3,4]$. Their risk of falling is 3 -fold higher compared to cognitively intact subjects [5]. When falling, they have a 3- to 4 -fold risk of severe fall-related injuries such as hip fractures [6]. People with dementia recover less well after a fall than those without dementia [7]. In view of the suffering caused by such falls, and the enormous cost of caring for people with dementia who have fallen, there is an urgent need to optimize the prevention of falls in this group.

Among various predictors for falls in the population with dementia (i.e. disease-specific motor impairment, type and severity of dementia, behavioral disturbances, functional impairment, and neuroleptics [8]), physical activity (PA) level has been identified as one important and potentially modifiable fall risk factor $[5,9,10]$. Some studies have found higher levels of PA to be protective against falling [9] whereas others have reported dementia-specific PA characteristics (i.e. wandering, agitated behavior) as fall predictors $[5,10]$. Existing fall-prediction studies in people with dementia have used subjective questionnaire-based PA assessment $[9,11]$ which may not allow accurate discrimination between 'protective' or 'risk' PA pattern. Objective monitoring of PA characteristics in person with dementia and exploration of their relationship with falling is needed to better understand and design effective interventions for this population [11].

In recent years, body-wearable sensor technology based on electromechanical sensors has provided a new avenue for objectively detecting and monitoring body motion and PA of individuals under natural conditions $[12,13]$. Wearable sensors have the benefits of portability and low cost, making these devices relevant to real-world fall risk assessment $[14,15]$. Since many falls occur in the home and community, where hazards are commonplace, it has been suggested to assess fall risk in these complex 'natural' environments [16, 17]. Further, there are significant concerns that people working in busy clinical settings do not have the time or equipment required to perform thorough objective fall risk assessments [18], and even where possible, these clinical settings do not emulate the natural home and community environment $[16,17]$. Additionally, fall risk assessment based on performancebased tests may be insensitive in those with cognitive impairment $[19,20]$. There is a need for simple clinical tools that can objectively assess fall risk in the rapidly growing population of cognitively impaired $[18,21]$. However, to date, there has been limited work performed in this important area [18, 21, 22].

A recent systematic review on wearable sensors-based fall prediction highlighted important shortcomings including (a) lack of prospective fall risk assessment, (b) lack of studies in more specialized high-risk populations such as those with dementia, and (c) lack of comparison between wearable sensor-based assessments and current clinical assessments for demonstrating benefits of the new sensor-based methods [22]. Importantly, the existing studies captured sensor data during in-clinic assessments such as the Timed Up and Go Test (TUG) [23, 24] or gait analysis [25] which require a specific test routine or laboratory setting. To our knowledge, no study explored the accuracy of sensor data captured in an everyday environment for prediction of future falls in people with dementia.

The aim of this study was to explore the validity of sensor-derived PA parameters quantified within a natural environment for predicting future falls in people with dementia. A second aim was to compare the validity of sensor-based fall risk assessment with conventional fall risk measures.

\section{Methods}

Sample

Participants were recruited from rehabilitation wards of a geriatric hospital (Agaplesion Bethanien Hospital, Heidelberg, Germany) at the end of rehabilitation. In individuals who met inclusion criteria for cognitive impairment (Mini-Mental State Examination, MMSE [26], score 17-26), a dementia diagnosis was confirmed according to international standards [27, 28]. Diagnosis was based on medical history, clinical examination, cerebral imaging, established neuropsychological test battery (Consortium to Establish a Registry for Alzheimer's Disease [29]), and the Trail Making Test [30]. Further inclusion criteria were written informed consent, approval by the legal guardian (if appointed), aged 65 and older, and no uncontrolled or terminal neurological, cardiovascular, metabolic, or psychiatric disorder. The study was approved by the Medical Department of the University of Heidelberg Ethics Committee in accordance with the Helsinki Declaration.

\section{Descriptive Measures}

Age, gender, cognitive performance (MMSE) [26], activities of daily living (ADL, Barthel Index) [31], fear of falling (Falls Efficacy Scale-International, FES-I) [32], depression (Cornell Scale for Depression in Dementia) [33], comorbidity (Cumulative Illness Rating Scale, CIRS) [34], and previous falls (in the last year, retrospective documentation) as obtained by self-report. 
Performance-Based Assessment of Functional Status

Performance-Oriented Mobility Assessment (POMA). The POMA [35] is a reliable and valid clinical test to assess gait and mobility deficits in specified motor tasks, related to risk of falling (i.e. rising from a chair, standing balance, turning, initiating gait, sitting down) in older adults and patient populations [36]. The total score range is $0-28$ with higher values indicating better performance. An experienced therapist instructed the participants how to perform the maneuvers, supervised the participants, and scored each participant's performance.

Timed Up and Go Test. The TUG [37] was used to test participants' basic functional mobility. The TUG is a reliable and valid clinical test to quantify mobility performance by timing participants with a stopwatch while rising from an armchair, walking $3 \mathrm{~m}$, turning, walking back, and sitting down.

5-Chair Stand. The 5-chair stand test is an established functional assessment in older adults, measuring the time (seconds) required to complete 5 repeated chair stands [38]. Participants were asked to stand up 5 times from the initial sitting position as quickly as possible.

\section{PA Assessment}

PA was quantified during a 24-hour period by a motion sensor (Physilog [13]) attached to the chest with an elastic belt. Patients were visited at home for attaching/detaching the sensor. All measures were conducted during a weekday. The Physilog system (BioAGM, CH) is a small $(95 \times 60 \times 22 \mathrm{~mm})$, light $(122 \mathrm{~g})$, long-term recording system containing inertial sensors (two accelerometers and one gyroscope) with software developed to identify postural positions and movements such as walking, standing, sitting, or lying $[12,13,15]$. A walking period was defined as an interval with at least 3 successive steps as described in the validation study of the Physilog [13]. Activities with $<3$ steps were considered as standing (e.g. working in the kitchen and moving $<3$ steps). The analysis algorithm is described elsewhere in detail [13]. It has proven to be sensitive (87-99\%) and specific (87-99.7\%) for detection of the PA pattern in different samples of older adults and patients [12, 13, 15, 39].

Nine PA parameters were calculated which represent characteristics of walking, standing, sitting and lying: (1) walking during $24 \mathrm{~h}, \%$; (2) average duration of all walking bouts conducted during the 24-hour measurement (=walking bout average duration), s; (3) duration of the longest walking bout (=longest walking bout duration), s; (4) variability of the duration of walking bout as calculated by the coefficient of variation $(\mathrm{CV})$ (=walking bout duration variability), \%; (5) standing during $24 \mathrm{~h}, \%$; (6) standing bout average duration, s; (7) sitting during $24 \mathrm{~h}, \%$; (8) sitting bout average duration, s, and (9) lying during $24 \mathrm{~h}, \%$.

\section{Assessment of Falls}

All study participants were monitored for falls for 3 months after the initial baseline assessment. Fall calendars were sent to the participants with written instructions and a prepaid return envelope to return the calendar every month. Phone calls were used to remind the participants of missing calendar fall logs. A fall was defined as 'an unexpected event in which the participants come to rest on the ground, floor, or lower level' [40]. Following a previous fall prediction study in people with dementia [19], the 3-month follow-up period was chosen in an attempt to be long enough to capture fall occurrences but not so long that the progression of the dementia could be a confounding factor.

Fall Prediction via Activity Monitoring

\section{Statistical Analysis}

Each participant was dichotomously categorized as a 'non-faller' or a 'faller' (at least one fall in a 3-month follow-up period). The means, SD and range were calculated, for non-fallers and fallers, for each of the variables reported in the present study. The MannWhitney $U$ test was used to evaluate the validity of variables to discriminate between non-fallers and fallers due to non-normal distribution of several continuous variables. $\chi^{2}$ tests were used for dichotomous variables.

Logistic regression analysis was employed to examine the relationship between each study variable and risk of falling. First, univariate logistic regression was employed to investigate the relationship of the test variables using 'faller/non-faller' as the dependent variable. This strategy reflects the exploratory character of the study. The odds ratio (OR) and coefficient of determination $\left(\mathrm{R}^{2}\right)$ were calculated for each explanatory variable. All variables were treated as continuous except 'previous faller' which was treated dichotomously (yes/no). Second, stepwise multivariate logistic regression, using the variables found to be significantly associated in the univariate analysis, was performed to investigate the independent effects of variables in predicting fallers. The receiver operating curve (ROC) and area under the curve (AUC) were calculated for different fall-prediction models. Sensitivities and specificities for different cutoff values were calculated for non-categorical variables shown to have an independent effect on predicting fallers. A two-sided $\mathrm{p} \leq 0.05$ was considered to be statistically significant. Statistical analysis was performed using SPSS statistics 21.0 (IBM, Armonk, N.Y., USA).

\section{Results}

118 people were asked to participate in the study. Of these, 115 (97.5\%) agreed to take part. The $3(2.5 \%)$ who declined did so because they did not like the idea of wearing the activity sensor. Another 6 participants (5.2\%) removed the activity sensor before the end of the 24-hour period and were excluded from the analysis.

77 participants $(67.0 \%)$ completed the study at 3 months. 3 died (2.6\%) and 29 (25.2\%) did not complete the calendar-based fall documentation and were excluded from the analysis. The sample population comprised older adults (age $81.8 \pm 6.3$ years) with impaired cognitive (MMSE score $22.1 \pm 3.2$ ) and functional (Barthel Index score 82.7 \pm 14.2 ; POMA score $21.0 \pm 4.5$ ) status. Participants had been discharged from a geriatric rehabilitation ward. Reasons for rehabilitation were: cerebrovascular diseases $15.7 \%$, lower limb fractures $13.7 \%$, other fracture $11.8 \%$, heart disease $11.8 \%$, and miscellaneous diagnoses including genitourinary, digestive, neoplasm, respiratory $47.0 \%$. During the time of PA assessment, 68 participants $(88.3 \%)$ were living independently at home, partly with supportive care, and $9(11.7 \%)$ were institutionalized. 28 participants (36.4\%) had fallen during the 3-month follow-up period. 
Table 1. Differences between fallers and non-fallers for descriptive variables, performance-based tests, and physical activity parameters

\begin{tabular}{|c|c|c|c|}
\hline Variable & Fallers $(\mathrm{n}=28)$ & Non-fallers $(n=49)$ & $\mathrm{p}$ value \\
\hline \multicolumn{4}{|l|}{ Descriptive variables } \\
\hline Age, years & $82.0 \pm 7.1$ & $81.8 \pm 5.9$ & 0.836 \\
\hline Male, $\%$ & 21.4 & 34.7 & 0.221 \\
\hline MMSE, score & $22.0 \pm 3.4$ & $22.1 \pm 3.1$ & 0.919 \\
\hline Barthel Activities of Daily Living, score & $81.6 \pm 16.4$ & $83.2 \pm 12.9$ & 0.991 \\
\hline Falls Efficacy Scale-International, score & $26.3 \pm 8.6$ & $27.0 \pm 8.7$ & 0.815 \\
\hline Cornell Scale for Depression, score & $7.0 \pm 4.7$ & $5.3 \pm 4.4$ & 0.069 \\
\hline Cumulative Illness Rating Scale, score & $24.0 \pm 3.6$ & $24.2 \pm 3.4$ & 0.682 \\
\hline Previous fall (last year), $\%$ & 75.0 & 42.9 & $0.006^{*}$ \\
\hline \multicolumn{4}{|l|}{ Living situation, $\%$} \\
\hline Community-dwelling & 85.7 & 89.8 & 0.592 \\
\hline Institutionalized & 14.3 & 10.2 & \\
\hline \multicolumn{4}{|l|}{ Performance-based tests } \\
\hline TUG, s & $13.3 \pm 5.9$ & $14.3 \pm 5.4$ & 0.236 \\
\hline Performance-Oriented Mobility Assessment, score & $21.0 \pm 4.3$ & $21.0 \pm 4.7$ & 0.928 \\
\hline 5-Chair stand, s & $15.9 \pm 6.9$ & $15.2 \pm 4.0$ & 0.553 \\
\hline \multicolumn{4}{|l|}{ Physical activity parameters } \\
\hline \multicolumn{4}{|l|}{ Walking } \\
\hline Walking during 24 h, \% & $4.1 \pm 3.1$ & $4.9 \pm 2.8$ & 0.117 \\
\hline Walking bout average duration, s & $10.7 \pm 2.3$ & $13.5 \pm 5.2$ & $0.008^{*}$ \\
\hline Longest walking bout duration, s & $89.9 \pm 100.2$ & $200.5 \pm 281.7$ & $0.009 *$ \\
\hline Walking bout duration variability, CV, \% & $87.1 \pm 35.5$ & $126.5 \pm 80.1$ & $0.027^{*}$ \\
\hline \multicolumn{4}{|l|}{ Standing } \\
\hline Standing during $24 \mathrm{~h}, \%$ & $14.0 \pm 7.9$ & $12.0 \pm 6.1$ & 0.403 \\
\hline Standing bout average duration, $s$ & $51.1 \pm 30.4$ & $40.8 \pm 11.9$ & $0.050 *$ \\
\hline \multicolumn{4}{|l|}{ Sitting } \\
\hline Sitting during $24 \mathrm{~h}, \%$ & $38.8 \pm 14.4$ & $39.5 \pm 10.5$ & 0.857 \\
\hline Sitting bout average duration, $s$ & $583.7 \pm 309.6$ & $618.4 \pm 314.8$ & 0.703 \\
\hline \multicolumn{4}{|l|}{ Lying } \\
\hline Lying during 24 h, \% & $43.1 \pm 10.2$ & $43.7 \pm 10.8$ & 0.983 \\
\hline
\end{tabular}

Validity of Variables to Discriminate between Fallers and Non-Fallers

Comparison of study variables between fallers and non-fallers are displayed in table 1. Fallers and non-fallers did not significantly differ for age, gender, cognitive status, ADL status, depression, comorbidities, or living situation (community-dwelling vs. institutionalized) $(\mathrm{p}=$ 0.069-0.991). Participants who fell during the 3 -month observation period had significantly fallen more often in the last year (fallers $75 \%$, non-fallers $42.9 \%$; $p=0.006$ ).

Surprisingly, no significant differences between fallers and non-fallers were obtained for performance-based tests $(\mathrm{p}=0.236-0.928)$. In contrast, significant differences between both groups were obtained for sensor-based PA parameters related to walking and standing. The 'walking bout average duration' was lower in fallers (mean $10.7 \pm 2.3 \mathrm{~s}$ ) compared to non-fallers (mean $13.5 \pm 5.2 \mathrm{~s}$, $\mathrm{p}=0.008$; fig. 1a). The 'longest walking bout duration' was shorter in fallers (mean $89.9 \pm 100.2 \mathrm{~s}$ ) compared to non-fallers (mean $200.5 \pm 281.7$ s, $p=0.009$; fig. 1b). The 'walking bout duration variability' was lower in fallers (CV mean $87.1 \pm 35.5 \%$ ) compared to non-fallers (CV mean $126.5 \pm 80.1 \%, p=0.027$; fig. 1c). Interestingly, fallers had a higher 'standing bout average duration' (mean $51.1 \pm 30.4 \mathrm{~s}$ ) compared to non-fallers (mean $40.8 \pm$ $11.9 \mathrm{~s}, \mathrm{p}=0.050$; fig. $1 \mathrm{~d})$.

\section{Predictor Variables for Falls}

In the univariate regression analysis, four variables were significantly associated with the risk of falling in the 
Fig. 1. Sensor-derived PA parameters related to walking $(\mathbf{a}-\mathbf{c})$ and standing $(\mathbf{d})$ discriminated between fallers and non-fallers with dementia (mean \pm standard error).

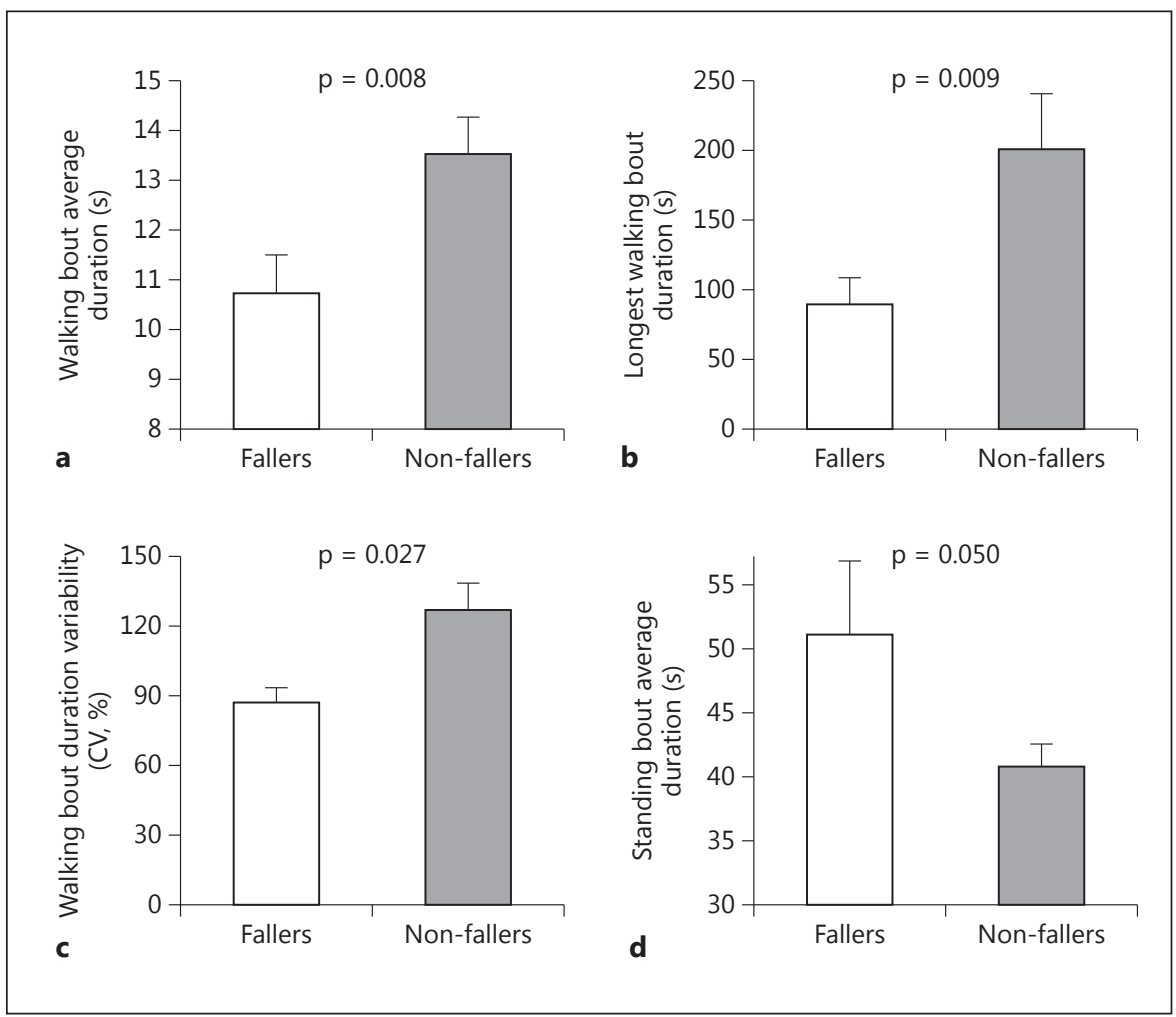

Table 2. Results of univariate logistic regression

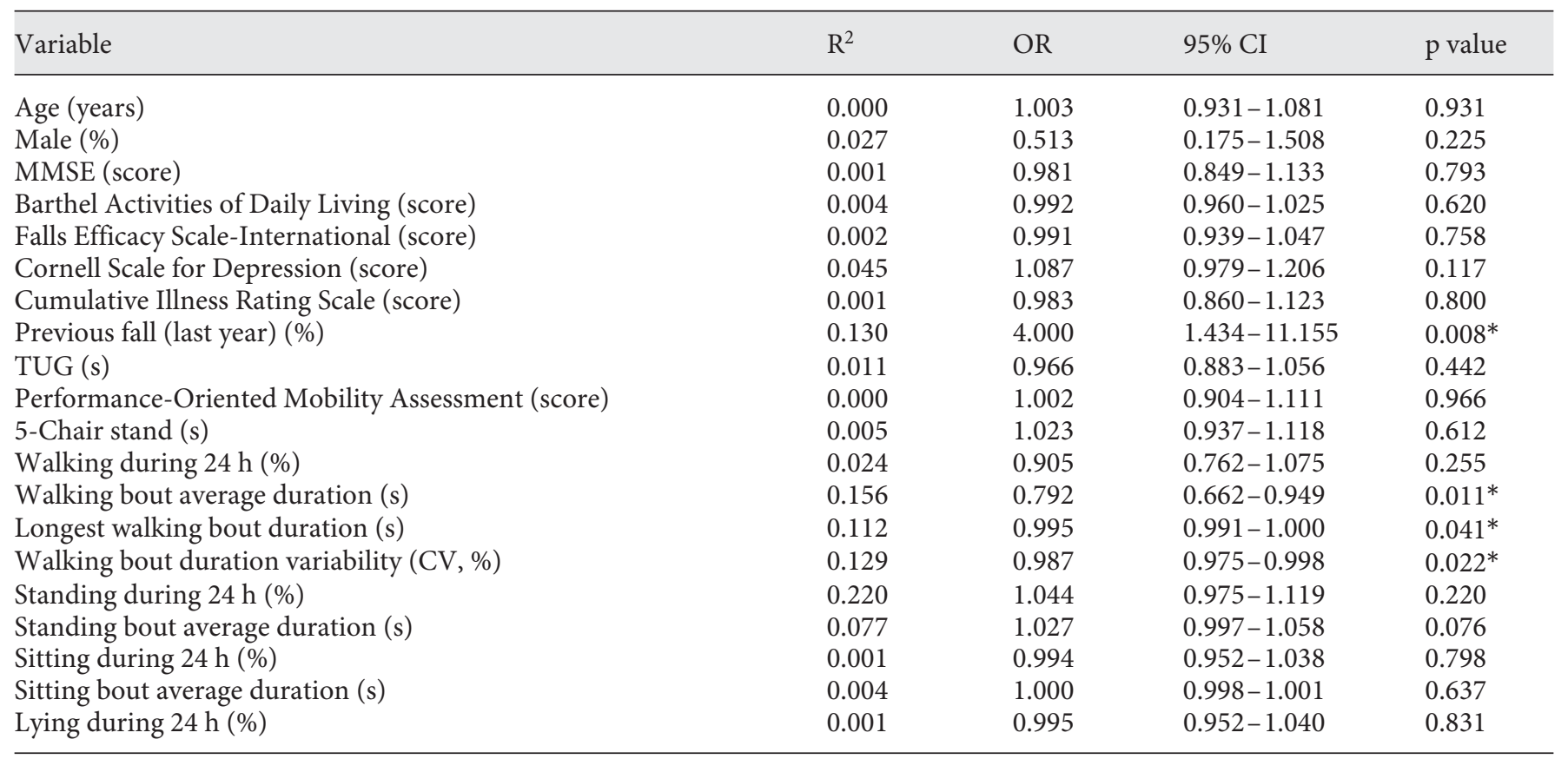

$\mathrm{CV}=$ Coefficient of variation. ${ }^{*} \mathrm{p} \leq 0.05$ 


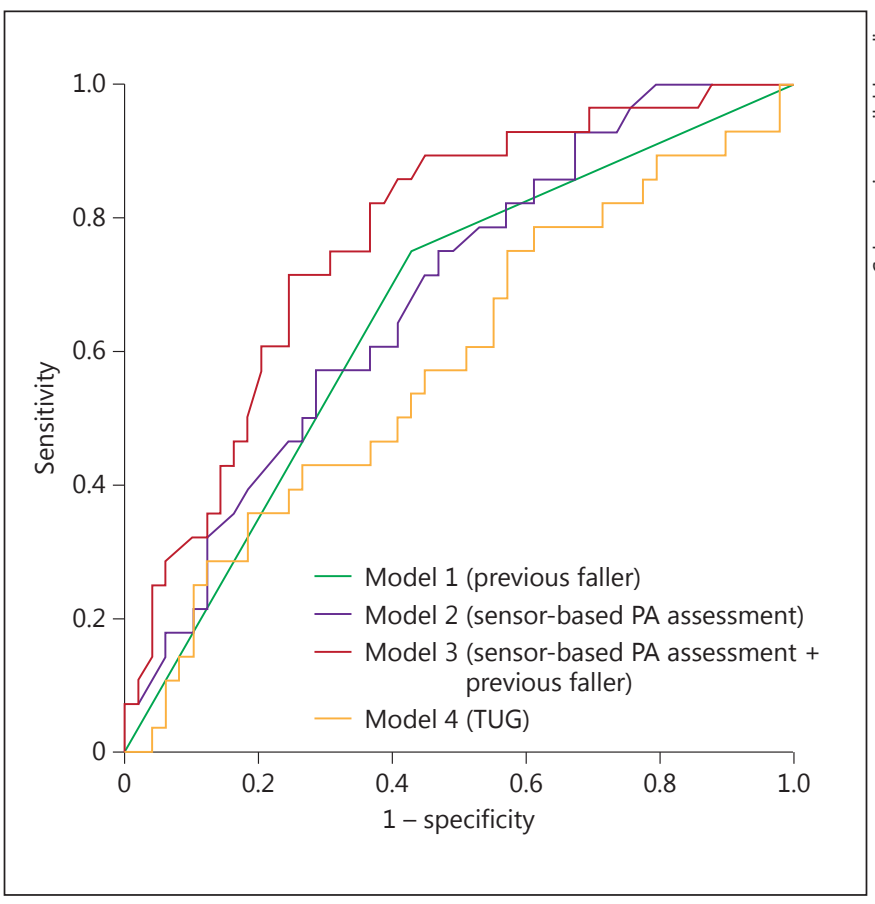

Fig. 2. ROCs of different models for predicting future falls in people with dementia: Model 1 uses 'previous faller' data (AUC 0.661), Model 2 uses the 'walking bout average duration' obtained by the PA sensor (AUC 0.684), Model 3 combines 'previous faller' and 'walking bout average duration' (AUC 0.771), and Model 4 uses TUG results (AUC 0.582).

Table 3. Sensitivities, specificities, OR of different cutoffs for the 'walking bout average duration'

\begin{tabular}{lllll}
\hline $\begin{array}{l}\text { Cutoffs, } \\
\text { s }\end{array}$ & $\begin{array}{l}\text { Sensitivity, } \\
\%\end{array}$ & $\begin{array}{l}\text { Specificity, } \\
\%\end{array}$ & OR $(95 \% \mathrm{CI})$ & p value \\
\hline 8 & 14.3 & 93.3 & $2.56(0.53-12.36)$ & 0.243 \\
9 & 21.4 & 87.8 & $1.96(0.56-6.77)$ & 0.291 \\
10 & 39.3 & 81.6 & $2.88(1.01-8.20)$ & 0.048 \\
11 & 60.7 & 61.2 & $2.44(0.94-6.32)$ & 0.066 \\
12 & 78.6 & 46.9 & $3.24(1.12-9.39)$ & 0.030 \\
13 & 82.1 & 38.8 & $2.91(0.95-8.97)$ & 0.062 \\
14 & 89.3 & 32.7 & $4.04(1.06-15.4)$ & 0.041 \\
15 & 92.9 & 32.7 & $6.30(1.33-29.91)$ & 0.020 \\
\hline
\end{tabular}

next 3 months: 'previous faller', 'walking bouts average duration', 'longest walking bout duration', and 'walking bout duration variability' (table 2). The best-fit model was found for 'walking bout average duration' $\left(\mathrm{R}^{2}=0.156\right)$.

Two variables, 'previous faller' (adjusted OR 4.44; 95\% CI 1.51-13.09; $\mathrm{p}=0.007$ ) and 'walking bout average dura- tion' (adjusted OR 0.79; 95\% CI 0.66-0.95; $\mathrm{p}=0.012$ ) remained in the multivariate model $\left(\mathrm{R}^{2}=0.276\right)$ suggesting that these two variables are independent predictors. We checked the multivariate logistic regression analysis using the 'methods = enter' methodology and the results were similar, with no other variable having an independent significant effect in predicting fallers.

Four models for prospective fall prediction were calculated. Model 1 using 'previous faller', Model 2 using 'walking bout average duration', and Model 3 using a combination of 'previous faller' and 'walking bout average duration'. Model 4 uses the TUG for comparing results of performance-based tests with the sensor-based fall risk assessment. The ROCs for the four models are displayed in figure 2 .

The AUC for Model 1 ('previous fallers') was 0.661 (95\% CI $0.535-0.786 ; \mathrm{p}=0.020)$ with a sensitivity of $75.0 \%$ and specificity $57.1 \%$ for predicting future falls.

The AUC for Model 2 ('walking bout average duration') was 0.684 (95\% CI 0.564-0.803; $\mathrm{p}=0.008)$. The sensitivities and specificities for different cutoff values for Model 2 are displayed in table 3. A cutoff of $15 \mathrm{~s}$ gives 93\% sensitivity, but low specificity (33\%). A cutoff of $8 \mathrm{~s}$ gives $93 \%$ specificity but the sensitivity is considerably reduced $(14.3 \%)$. The OR for predicting fallers ranged between 1.96 and 6.30 depending on the cutoff value (table 3).

The highest AUC $(0.771 ; 95 \%$ CI $0.664-0.878$; $\mathrm{p} \leq$ 0.001 ) was obtained by Model 3 combining 'previous faller' and 'walking bout average duration'.

Using a cutoff value for 'walking bout average duration' $<15 \mathrm{~s}$ combined with a previous history of falls, the sensitivity was $71.5 \%$ and the specificity $75.5 \%$. The OR for experiencing a fall in the following 3 month was 7.71 (95\% CI 2.71-21.96; $\mathrm{p}<0.001$ ).

The lowest AUC (0.582; 95\% CI 0.447-0.716; $\mathrm{p}=$ 0.236) was obtained for Model 4 using the TUG.

\section{Discussion}

This study evaluated the discriminative and predictive validity of sensor-derived PA parameters for identifying future falls in people with confirmed mild to moderate dementia. Present results suggest that traditional performance-based tests are insensitive predictors of fall risk, whereas PA parameters related to walking and standing are useful fall risk indicators. To our knowledge, this is the first study which used objective PA monitoring for predicting falls in people with dementia. Our findings 
suggest that analysis of everyday motions using wearable sensors can enhance accuracy of traditional fall risk assessment in high-risk populations such as people with dementia.

\section{Validity of Variables to Discriminate between Fallers} and Non-Fallers

None of the demographic data, performance-based tests, and questionnaires, except 'previous fallers', discriminated between future fallers and non-fallers. Our findings confirm results of a previous study in people with dementia in which only previous falls but not performance-based tests and demographic data did predict future falls (4-month follow-up period) [19]. Reliability of performance-based tests can be affected by dementiaassociated symptoms such as impaired executive function, memory, and attention [20], which may explain insufficient validity of these measures for predicting falls. But even in cognitively intact older adults, performancebased tests such as TUG may have only poor to moderate accuracy for predicting future falls as highlighted in a recent systematic review [41].

Results of this study demonstrate that fallers and nonfallers differ in PA pattern. On the same note, the present results suggest that measuring the overall daily walking time is not an accurate parameter for discriminating between fallers and non-fallers. In contrast, specific walking characteristics such as the duration of walking bouts were found to be sensitive discriminators. Interestingly, some PA characteristics were protective (long walking bouts) whereas others increased risk of falling (long standing bouts). The longest bout walked during the 24-hour measurement was only half as long in fallers compared to non-fallers and a sensitive discriminator. On average, duration of walking bouts was significantly shorter in fallers compared to non-fallers.

PA behavior is affected by personal, social, and environmental factors [42]. We can only speculate about the factors accounting for the differences in PA characteristics between both groups. Differences were not related to sociodemographics or clinical status. Importantly, functional performances as quantified by the tests used in this study did not explain the differences in walking characteristics. A poor relationship between functional performances and PA level in older adults has been reported previously [43].

Our findings suggest that fallers had a more interrupted walking pattern, with short walking bouts rather than continuously long walking bouts. Results could indicate less direct and more inefficient travel pattern in fallers, potentially related to disorientation or wandering behavior (i.e. random travel and pacing) as described in previous studies [44]. Also, dementia-associated dual-task deficits (i.e. limited ability to walk with a concurrent task [45]) may have accounted for shorter walking bouts found in fallers.

The lack of long walking bouts in the daily activity profile of fallers may also indicate limited outdoor walking. Fallers may have walked predominately in indoor spaces as indicated by shorter walking bouts. Results may suggest that more fallers were housebound when compared to non-fallers, potentially due to environmental barriers (i.e. inability to climb stairs) or lack of support for outdoor or longer range activities by a caregiver/relative. Being housebound and $>75$ years of age has been previously identified as a risk factor of falling indoors [46].

The shorter walking bouts found in fallers in the present study could be related to previous falls. Future fallers had significantly more previous falls, as found in previous studies in people with dementia $[9,19]$. In the present study, previous falls may have caused changes in walking characteristics, potentially due to fear of falling. Since we did not find any differences in fear of falling between fallers and non-fallers based on self-report (FES-I), our results may indicate differences in self-report and observed functioning (walking) as reported in previous studies [47].

The variability in duration of all walking bouts in $24 \mathrm{~h}$ was significantly higher in non-fallers compared to fallers and was identified as a sensitive discriminative parameter. The increased variability indicates that non-fallers had a more diverse PA pattern including both short and long walking bouts over the course of the day. A diversity of activities has been previously described as protective against falls [46].

Interestingly, fallers had significantly longer standing bouts compared to non-fallers. As per algorithm, standing includes phases of standing as well as walking less than 3 steps. Walking a few steps and standing again could indicate fidgety, restlessness and agitation as common dementia-associated behavioral symptoms, which have been linked to increased fall risk [8]. Subtle dementia-associated impairments in postural control [48], not detected by the performance-based test, may explain increased fall risk during phases of prolonged standing and fidgeting as obtained in the present study. Our results may indicate that such fall risk-related activity behavior could be quantified in an everyday environment using wearable sensors. 
For individuals who are already fall-prone, increased activity may result in a greater risk of falling due to increased exposure to environmental hazards [49]. If walking is considered as the 'exposure' to fall risk in our study, results may indicate that fallers had less exposure and yet they still fell more. This may suggest that specific PA pattern such as short walking bouts or prolonged phases of standing are more sensitive indicators of fall risk, compared to estimating exposure by overall time of walking.

\section{Predictor Variables for Falls}

The results of the regression analysis suggest that, out of the various PA variables examined, the 'walking bout average duration' performed the best in predicting falls in older adults with dementia. Each second of shorter 'walking bout average duration' was associated with a $26 \%$ increased chance of becoming a faller. Someone with a 'walking bout average duration' $>15 \mathrm{~s}$ is very unlikely to fall. Someone with a 'walking bout average duration' $<15 \mathrm{~s}$ had a 6.3 times increased fall chance compared to someone above this threshold. Using this cutoff, the sensitivity is $93 \%$; however, the specificity is only $33 \%$, implying that it will predict most of the future falls, but will falsely predict falls in $77 \%$ of non-fallers. Thus, while this variable is not a stand-alone candidate for fall prediction, it could add precision to a fall index.

Combining the independent predictors 'walking bout average duration' and 'previous faller' improved fall prediction to a clinically useful level. A 'walking bout average duration' $<15$ s combined with a previous history of falls gave a sensitivity of $72 \%$ and a specificity of $76 \%$. Intervening on these individuals would represent reasonable targeting as only $24 \%$ of people measured at high risk would not have subsequently fallen.

Only a few studies sought to prospectively predict falls using wearable sensors [23, 24]. Marschollek et al. [24] followed up 50 geriatric patients for 1 year after instrumented TUG and gait assessment. In that study, an AUC of 0.65 was reported based on accelerometer-derived parameters classified using logistic regression [24], which is comparable with our fall prediction Model 2 using sensor data only ('walking bout average duration', AUC 0.68). Interestingly, in the study of Marschollek et al., predictive performance was increased when accelerometer data were combined with PA questionnaire data (AUC 0.72), whereas a high activity level was associated with low fall risk. Predictive validity of this combined model is comparable with our Model 3 combining sensor data and questionnaire data ('previous faller') (AUC 0.77).
Greene et al. [23] reported a good validity (AUC 0.78) of instrumented TUG assessment for predicting future falls ( 2 years' follow-up) in community-dwelling older adults without cognitive impairment. Future studies need to investigate if similar results can be achieved in people with dementia.

In the present study, univariate analysis showed that some of the other PA variables ('longest walking bout duration', 'walking bout duration variability') also had value in predicting falls, although they were inferior to the 'walking bout average duration'. The 'walking bout average duration' includes elements of the other PA parameters studied. Someone walking long distances over the course of the day increases the 'walking bout average duration' while walking both short distances and long distances increases the 'walking bout duration variability'. A high degree of correlation and co-linearity therefore would be expected between these PA parameters, which is why on multivariable analysis, the other PA were no longer independent predictors.

\section{Limitations and Future Directions}

One obvious limitation of this study is the small sample size. However, we feel that this limitation does not invalidate our findings, given that the main aim of the study was to explore the association between PA pattern and future falling. Our proposed models must be validated in a larger sample size to evaluate their true predictive potential.

The battery life of the activity monitor used in the present study restricted the monitoring period to $24 \mathrm{~h}$. This assessment period did not cover day-to-day variability in PA, although PA behavior in older adults is less variable than in younger populations [50] and day-today reliability of PA assessment was high in a sample of older adults ( $>60$ years) [50]. The 24 -hour monitoring in our study may therefore have been sufficient to document habitual PA because of low day-to-day variability. However, further research should address whether a longer period of monitoring increases the accuracy of fall prediction.

Increased standing bout duration was identified as a fall risk factor in the present study. As a limitation, the algorithm used in this study cannot discriminate between phases of quiet standing and walking very short bouts ( $<3$ steps). Further algorithm development could separate these phases to better understand their association with fall risk.

While we have identified novel objective fall-associated PA parameters, further studies are required to eluci- 
date their biopsychosocial interpretation in the context of fall risk assessment. Dementia-specific behavioral symptoms such as wandering or agitation should be assessed by standardized instruments $[51,52]$ for examining their association with the fall risk-related PA pattern found in this study. More accurate assessments including spatiotemporal gait analysis [25] and dual-task assessment [45] should be used for measuring dementia-specific motorcognitive deficits, potentially accounting for the fall riskrelated PA pattern found in this study (i.e. short walking bouts). Further, the association between fall risk-related PA behavior and environmental barriers in the home and immediate outdoor environment need to be quantified in future studies, for instance by using the Housing Enabler instrument [53].

In our study, accuracy of the reported level of fear of falling (FES-I) may have been influenced by difficulty in comprehending questions or reporting on subjective states, as discussed previously [54]. Future studies may use the Iconographical Falls Efficacy Scale using pictures as visual cues, which has previously been validated in the cognitively impaired [54].

We observed a high rate of falling (36.4\% of subjects) during a relatively brief follow-up (3 months). Future studies should investigate whether non-fallers as identified by the presented short-term fall prediction approach become fallers during a longer follow-up period.

\section{Conclusion}

We found that the combination of PA monitoring and fall history has the potential to provide a clinically meaningful surveillance of people with dementia at high risk of falling. This information could be used to provide targeted fall prevention interventions. Present findings may help to design mHealth technologies using monitoring of everyday activities for the purpose of fall risk assessment in people with dementia.

\section{Acknowledgements}

This study was partially supported by an STTR-Phase II Grant (Award No. 2R42AG032748) from the National Institute on Aging, a postdoctoral research fellowship of the German Academic Exchange Service (DAAD), the Baden-Württemberg Stiftung, the Robert Bosch Stiftung, and the Dietmar Hopp Stiftung. The content is solely the responsibility of the authors and does not necessarily represent the official views of the funding institutions. We thank Marilyn Gilbert for critical revision of the manuscript (Interdisciplinary Consortium on Advanced Motion Performance, University of Arizona).

\section{Disclosure Statement}

The authors have no conflicts of interest to disclose.

\section{References}

1 Tinetti ME, Inouye SK, Gill TM, Doucette JT: Shared risk factors for falls, incontinence, and functional dependence. Unifying the approach to geriatric syndromes. JAMA 1995; 273:1348-1353.

2 Tinetti ME, Williams CS: Falls, injuries due to falls, and the risk of admission to a nursing home. N Engl J Med 1997;337:1279-1284.

-3 Morris JC, Rubin EH, Morris EJ, Mandel SA: Senile dementia of the Alzheimer's type: an important risk factor for serious falls. J Gerontol 1987;42:412-417.

-4 Van Doorn C, Gruber囚Baldini AL, Zimmerman S, Richard Hebel J, Port CL, Baumgarten M, Quinn CC, Taler G, May C, Magaziner J: Dementia as a risk factor for falls and fall injuries among nursing home residents. J Am Geriatr Soc 2003;51:1213-1218.

5 Buchner DM, Larson EB: Falls and fractures in patients with Alzheimer-type dementia. JAMA 1987;257:1492-1495.

6 Lord SR, Sherrington C, Menz HB: Falls in Older People. Cambridge, Cambridge University Press, 2001.
Shaw FE: Falls in cognitive impairment and dementia. Clin Geriatr Med 2002;18:159173.

>8 Härlein J, Dassen T, Halfens RJ, Heinze C: Fall risk factors in older people with dementia or cognitive impairment: a systematic review. J Adv Nurs 2009;65:922-933.

-9 Allan LM, Ballard CG, Rowan EN, Kenny RA: Incidence and prediction of falls in dementia: a prospective study in older people. PLoS One 2009;4:e5521.

10 Katz IR, Rupnow M, Kozma C, Schneider L: Risperidone and falls in ambulatory nursing home residents with dementia and psychosis or agitation: secondary analysis of a doubleblind, placebo-controlled trial. Am J Geriatr Psychiatry 2004;12:499-508.

11 Suttanon P, Hill KD, Said CM, Dodd KJ: A longitudinal study of change in falls risk and balance and mobility in healthy older people and people with Alzheimer disease. Am J Phys Med Rehabil 2013;92:676-685.
12 Najafi B, Armstrong DG, Mohler J: Novel wearable technology for assessing spontaneous daily physical activity and risk of falling in older adults with diabetes. J Diabetes Sci Technol 2013;7:1147-1160.

13 Najafi B, Aminian K, Paraschiv-Ionescu A, Loew F, Bula CJ, Robert P: Ambulatory system for human motion analysis using a kinematic sensor: monitoring of daily physical activity in the elderly. IEEE Trans Biomed Eng 2003;50:711-723.

14 Aminian K, Najafi B: Capturing human motion using body-fixed sensors: outdoor measurement and clinical applications. Comput Anim Virtual Worlds 2004;15:79-94.

15 Najafi B, Aminian K, Loew F, Blanc Y, Robert PA: Measurement of stand-sit and sit-stand transitions using a miniature gyroscope and its application in fall risk evaluation in the elderly. IEEE Trans Biomed Eng 2002;49:843-851.

16 Lord SR, Menz HB, Sherrington C: Home environment risk factors for falls in older people and the efficacy of home modifications. Age Ageing 2006;35:ii55-ii59. 
$\checkmark 17$ Weiss A, Brozgol M, Dorfman M, Herman T, Shema S, Giladi N, Hausdorff JM: Does the evaluation of gait quality during daily life provide insight into fall risk? A novel approach using 3-day accelerometer recordings. Neurorehabil Neural Repair 2013;27:742-752.

-18 Shany T, Redmond SJ, Marschollek M, Lovell $\mathrm{NH}$ : Assessing fall risk using wearable sensors: a practical discussion. A review of the practicalities and challenges associated with the use of wearable sensors for quantification of fall risk in older people. Z Gerontol Geriatr 2012;45:694-706.

19 Farrell MK, Rutt RA, Lusardi MM, Williams AK: Are scores on the physical performance test useful in determination of risk of future falls in individuals with dementia? J Geriatr Phys Ther 2011;34:57-63.

20 Hauer K, Oster P: Measuring functional performance in persons with dementia. J Am Geriatr Soc 2008;56:949-950.

21 Shany T, Redmond SJ, Narayanan MR, Lovell $\mathrm{NH}$ : Sensors-based wearable systems for monitoring of human movement and falls. IEEE Sens J 2012;12:658-670.

22 Howcroft J, Kofman J, Lemaire ED: Review of fall risk assessment in geriatric populations using inertial sensors. J Neuroeng Rehabil 2013;10:91.

23 Greene BR, Doheny EP, Walsh C, Cunningham C, Crosby L, Kenny RA: Evaluation of falls risk in community-dwelling older adults using body-worn sensors. Gerontology 2012; 58:472-480.

-24 Marschollek M, Rehwald A, Wolf K, Gietzelt M, Nemitz G, Meyer zu Schwabedissen H, Haux R: Sensor-based fall risk assessment an expert 'to go'. Methods Inf Med 2011;50: 420.

-25 Senden R, Savelberg H, Grimm B, Heyligers I, Meijer K: Accelerometry-based gait analysis, an additional objective approach to screen subjects at risk for falling. Gait Posture 2012; 36:296-300

26 Folstein MF, Folstein SE, McHugh PR: 'Minimental state'. A practical method for grading the cognitive state of patients for the clinician. J Psychiatr Res 1975;12:189-198.

-27 McKhann G, Drachman D, Folstein M, Katzman R, Price D, Stadlan EM: Clinical diagnosis of Alzheimer's disease: report of the NINCDS-ADRDA Work Group under the auspices of Department of Health and $\mathrm{Hu}$ man Services Task Force on Alzheimer's Disease. Neurology 1984;34:939-944.

-28 Roman GC, Tatemichi TK, Erkinjuntti T, Cummings JL, Masdeu JC, Garcia JH, Amaducci L, Orgogozo JM, Brun A, Hofman A, et al: Vascular dementia: diagnostic criteria for research studies. Report of the NINDS-AIREN International Workshop. Neurology 1993;43:250-260.
29 Morris JC, Mohs RC, Rogers H, Fillenbaum G, Heyman A: Consortium to establish a registry for Alzheimer's disease (CERAD) clini$\mathrm{cal}$ and neuropsychological assessment of Alzheimer's disease. Psychopharmacol Bull 1988;24:641-652.

30 Oswald WD: Nürnberger Altersinventar (NAI). Göttingen, Hogrefe, 1995.

31 Mahoney FI, Barthel DW: Functional evaluation: the Barthel Index. Md State Med J 1965; 14:61-65.

32 Yardley L, Beyer N, Hauer K, Kempen G, Piot-Ziegler C, Todd C: Development and initial validation of the Falls Efficacy Scale-International (FES-I). Age Ageing 2005;34:614619.

33 Alexopoulos GS, Abrams RC, Young RC, Shamoian CA: Cornell Scale for Depression in Dementia. Biol Psychiatry 1988;23:271284.

34 Parmelee PA, Thuras PD, Katz IR, Lawton MP: Validation of the cumulative illness rating scale in a geriatric residential population. J Am Geriatr Soc 1995;43:130-137.

35 Tinetti ME: Performance-oriented assessment of mobility problems in elderly patients. J Am Geriatr Soc 1986;34:119-126.

- 36 Kegelmeyer DA, Kloos AD, Thomas KM, Kostyk SK: Reliability and validity of the $\mathrm{Ti}$ netti Mobility Test for individuals with Parkinson disease. Phys Ther 2007;87:13691378.

-37 Podsiadlo D, Richardson S: The timed 'up \& go': a test of basic functional mobility for frail elderly persons. J Am Geriatr Soc 1991;39: 142-148.

38 Guralnik J, Simonsick E, Ferrucci L, Glynn R, Berkman L, Blazer D, Scherr P, Wallace R: A short physical performance battery assessing lower extremity function: association with self-reported disability and prediction of mortality and nursing home admission. J Gerontol 1994;49:M85-M94.

- 39 Salarian A, Russmann H, Vingerhoets FJ, Burkhard PR, Aminian K: Ambulatory monitoring of physical activities in patients with Parkinson's disease. IEEE Trans Biomed Eng 2007;54:2296-2299.

40 Hauer K, Lamb SE, Jorstad EC, Todd C, Becker C: Systematic review of definitions and methods of measuring falls in randomised controlled fall prevention trials. Age Ageing 2006;35:5-10.

41 Schoene D, Wu SM, Mikolaizak AS, Menant JC, Smith ST, Delbaere K, Lord SR: Discriminative ability and predictive validity of the timed up and go test in identifying older people who fall: systematic review and meta-analysis. J Am Geriatr Soc 2013;61:202-208.
42 Benzinger P, Iwarsson S, Kroog A, Beische D, Lindemann U, Klenk J, Becker C: The association between the home environment and physical activity in community-dwelling older adults. Aging Clin Exp Res 2014:1-9.

$\checkmark 43$ Nicolai S, Benzinger P, Skelton DA, Aminian $\mathrm{K}$, Becker C, Lindemann U: Day-to-day variability of physical activity of older adults living in the community. J Aging Phys Act 2010; 18:75-86.

44 Martino-Saltzman D, Blasch BB, Morris RD, McNeal LW: Travel behavior of nursing home residents perceived as wanderers and non-wanderers. Gerontologist 1991;31:666672.

45 Camicioli R, Howieson D, Lehman S, Kaye J: Talking while walking the effect of a dual task in aging and Alzheimer's disease. Neurology 1997;48:955-958.

46 Bath PA, Morgan K: Differential risk factor profiles for indoor and outdoor falls in older people living at home in Nottingham, UK. Eur J Epidemiol 1999;15:65-73.

-47 Feuering R, Vered E, Kushnir T, Jette AM, Melzer I: Differences between self-reported and observed physical functioning in independent older adults. Disabil Rehabil 2013, Epup ahead of print.

48 Franssen EH, Souren L, Torossian CL, Reisberg B: Equilibrium and limb coordination in mild cognitive impairment and mild Alzheimer's disease. J Am Geriatr Soc 1999;47: 463.

49 Tinetti ME: Prevention of falls and fall injuries in elderly persons: a research agenda. Prev Med 1994;23:756-762.

50 Rowe DA, Kemble CD, Robinson TS, Mahar MT: Daily walking in older adults: day-to-day variability and criterion-referenced validity of total daily step counts. J Phys Act Health 2007; 4:434-446.

51 Algase DL, Beattie ER, Bogue E-L, Yao L: The Algase Wandering Scale: initial psychometrics of a new caregiver reporting tool. Am J Alzheimers Dis Other Demen 2001;16:141152.

52 Cohen-Mansfield J, Marx MS, Rosenthal AS: A description of agitation in a nursing home. J Gerontol 1989;44:M77-M84.

53 Iwarsson S, Slaug B: The Housing Enabler. An Instrument for Assessing and Analysing Accessibility Problems in Housing. Nävlinge \& Staffanstorp, Veten \& Skapen HB \& Slaug Data Management, 2001.

54 Delbaere K, Close JCT, Taylor M, Wesson J, Lord SR: Validation of the Iconographical Falls Efficacy Scale in cognitively impaired older people. J Gerontol A Biol Sci Med Sci 2013;68:1098-1102. 